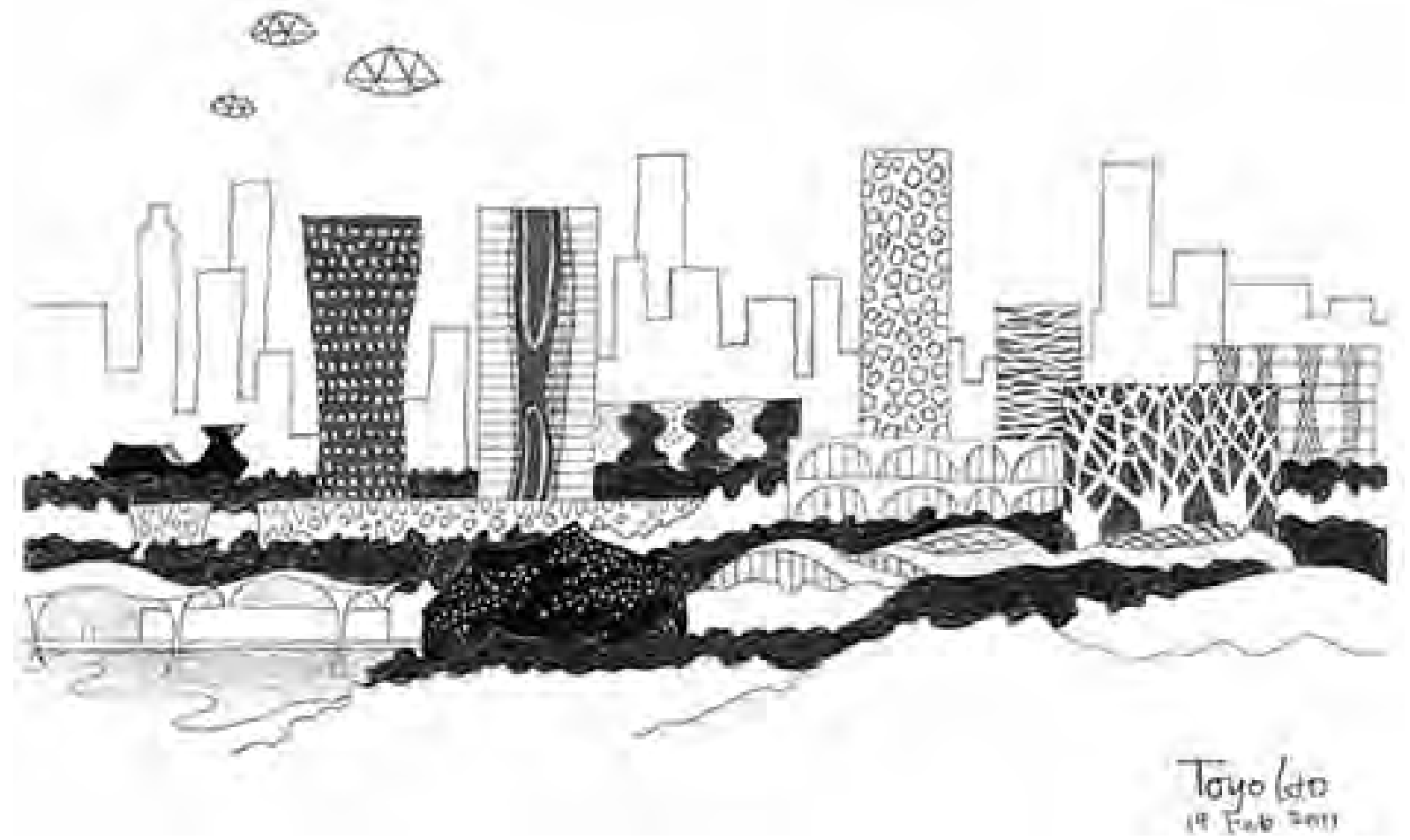




\section{Naturalización del espacio arquitectónico. Tres posiciones críticas alejadas del naturalismo Javier de Esteban}

El artículo analiza tres propuestas coincidentes en fechas, desarrolladas durante el cambio de siglo, que profundizan en la relación entre naturaleza y espacio arquitectónico. En los tres casos la interpretación de la naturaleza, entendida como constructo cultural, se integra en la propia concepción e ideación arquitectónica. Bajo lo que podríamos entender como un proceso de 'naturalización', estas prácticas tratan de superar aquellas autoproclamadas sostenibles o naturalistas, por lo general de poco alcance, recuperando algunas tradiciones y compartiendo una actitud crítica que nos permite repensar nuevas formas de visualizar la naturaleza y su relación con la arquitectura.

\section{PALABRAS CLAVE}

Naturalización, espacio arquitectónico, mediateca Sendai, Stortoget Kalmar, "The Mediated Motion"

\section{KEYWORDS}

Naturalisation, Architectural Space, Sendai Mediatheque, Stortoget Kalmar, "The Mediated Motion"

\section{NATURALIZACIÓN} FRENTE A NATURALISMO

“¿Qué es lo que conozco? ¿Es naturaleza? Una naturaleza tal
no tiene una esencia 'real'; no hay ningún secreto verdadero
que desvelar. Nunca me he aproximado a nada esencial que
no sea yo mismo y, además, de todos modos, ¿no es la
naturaleza un estado cultural? Lo que he llegado a conocer
mejor es mi propia relación con la llamada naturaleza (es decir, mi capacidad de orientarme en este espacio particular), mi capacidad de ver, sentir y moverme por los paisajes que me rodean. Al mirar la naturaleza no encuentro nada..., solo mi propia relación, aspectos de mi relación con los espacios. Vemos la naturaleza con ojos cultos. De nuevo, no existe ninguna naturaleza verdadera; solo existe nuestro constructo como tal. Con el simple hecho de mirar la naturaleza, la cultivamos en una imagen"'.

\begin{abstract}
Javier de Esteban Garbayo
U. P. Arquitectura, Universidad de Zaragoza Doctor Arquitecto por la UPM (2016). Obtuvo los títulos de Arquitecto por la Universidad de Navarra (2009) y Máster en Proyectos Arquitectónicos Avanzados por la UPM (2011). Profesor Asociado en la EINA de Zaragoza (2018) y Profesor Invitado en el Máster de Proyectos Arquitectónicos de la ETSAM (2014-2018), también ha sido Profesor Asociado en la ETSA de Valladolid (2016-2017) y Profesor Asistente en la ETSAM (2009-2015). Además ha sido Investigador en el Departamento de Arquitectura de la Universidad de Cambridge (2012-2013), Investigador contratado en el Grupo de Investigación en Vivienda Colectiva de la UPM (2010-2011) y acreditado como Profesor Ayudante Doctor por la ANECA. Fundador del estudio MydE Arquitectos.
\end{abstract}

Fig. 01

Idea de ciudad; donde medio natural $y$ construido forman una unidad indivisible que favorece la fluidez y la intercambiabilidad. Croquis, Toyo Ito, 19 febrero 2011. 
El artista estadounidense Robert Smithson describía la naturaleza como "simplemente otra ficción de los siglos XVIII y XIX". Esta provocadora afirmación venía a decir que la naturaleza debía entenderse como constructo cultural, hacia la cual el ser humano ha desplegado una mirada distinta a lo largo de los diversos periodos históricos. Tal y como se puede interpretar en obras como la icónica Spiral Jetty, Smithson trata de superar numerosas convenciones profundizando en la relación entre tecnología y formas naturales. El artista pone de relieve que la naturaleza no puede desligarse de su propia interpretación, mostrando un marco operativo de gran interés fundamentado en los cambios tecnológicos y sus posibilidades en la visualización y la comprensión de ésta ${ }^{2}$.

Esta concepción adquiere mayor relevancia si pensamos que la interpretación de la naturaleza ha cambiado sustancialmente a lo largo del siglo XX. En la actualidad parece estar moldeada por una conciencia medioambiental que ha ido en aumento desde los años setenta reflejada en publicaciones como Los límites del crecimiento y el uso habitual de términos como crisis energética o cambio climático. Así, la visión de la naturaleza como algo obvio, dado, coherente e inagotable, parece haberse desvanecido. Nos encontramos en una situación en la que las perspectivas globales han progresado considerablemente en nuestras vidas cotidianas, haciendo imprescindible tener que pensar en las consecuencias medioambientales de nuestras acciones individuales. En este sentido, parece pertinente preguntarnos acerca de cómo poder integrar estos temas en nuestras concepciones estéticas.

Si observamos la concepción de la naturaleza a lo largo de la modernidad, parece haberse alimentado constantemente la diferencia entre lo preindustrial y lo industrial, entre la naturaleza y los productos desarrollados por el ser humano, bien sean específicos de la industria o cualquier entorno construido. Casos, como la concepción de Central Park por parte de Frederick Law Olmsted resultan paradigmáticos. En el texto "Public Parks and the Enlargement of Towns" de 1870, Olmsted entiende el parque como algo ligado a la naturaleza, como un foco de salubridad (aludiendo al higienismo social) en contraposición a la ciudad y el medio urbano. Este hecho también se evidencia en los proyectos urbanos de Le Corbusier, como sucede en Ville Radieuse (1922), donde las torres de viviendas salpican una naturaleza ininterrumpida. El quinto punto para la nueva arquitectura, la terraza-jardín, también alimenta esta dualidad, concebido desde la devolución a la naturaleza del espacio ocupado por la huella del edificio.

Pero quizá el caso del Crystal Palace resulta más revelador en parte por lo que el propio edificio representa en la concepción moderna. En este caso, Joseph Paxton tuvo que preservar una hilera de olmos centenarios para que el barrio aristócrata de Hyde Park le concediera el permiso para llevar a cabo el pabellón de la Gran Exposición de Londres de 1951. Así, el edificio, que representa los avances de la industrialización tanto en el usos de los nuevos materiales (vidrio y acero) como en su propia construcción y ejecución (ensamblado por un ejército de trabajadores del ferrocarril en cuatro meses), incorpora la propia naturaleza que había expulsado como imagen (fig. 02).

El historiador y crítico Philip Ursprung denomina a esta actitud, o ideología, "naturalista", fundamentada "en la asunción de que en 


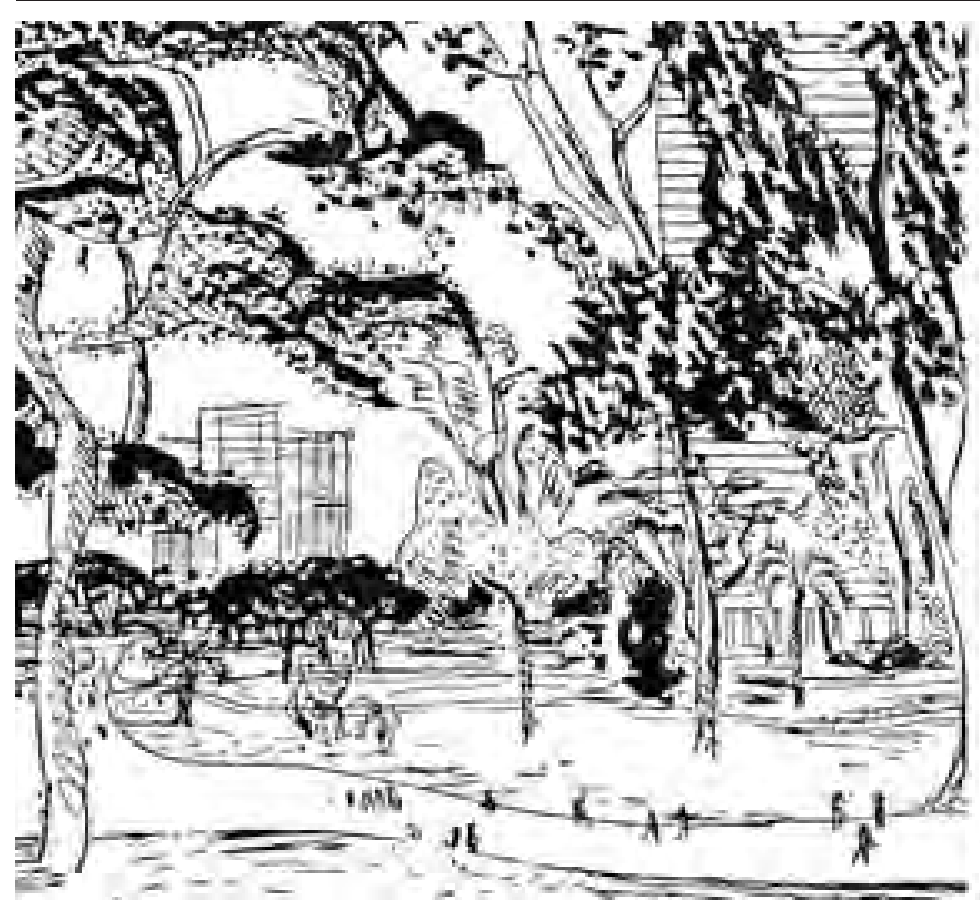

02

realidad existe una cosa, la naturaleza, y que el significado de la arquitectura reside en su capacidad de articular, enmarcar, ensalzar y controlar las fuerzas de la naturaleza". Ursprung propone superar esta dualidad alimentada en la modernidad "al igual que se hizo difícil distinguir entre pared y techo o entre interior y exterior". Añade que "la naturaleza y todos los emblemas por los que solía estar representada están completamente absorbidos en la actual esfera espacial"3.

Si retomamos la idea de Robert Smithson de entender la naturaleza como ficción, bien sea desde la noción de integración o desde su existencia como algo independiente, entonces habría que reformular la cuestión de cómo la naturaleza afecta y conforma el entorno construido por el ser humano. Ante la posibilidad de que la naturaleza pueda representarse en forma de imagen, texto o sistema de símbolos, surge también la posibilidad de que pueda alterarse y manipularse. Dentro de esta óptica, la idea de 'naturalización' se aleja del entendimiento de la naturaleza como algo dado o autónomo al entorno construido, para asumirla como proyección del ser humano y consustancial al propio diseño.

El desarrollo de una arquitectura como reflexión de un modo de concebir la naturaleza, que plantee una visualización específica de ésta, se muestra como vía a explorar, más aún dada la relevancia que la conciencia medioambiental ha adquirido en las últimas décadas. Sobre las dimensiones estéticas de esta conciencia, Iñaki Ábalos señala que "solo si hay una verdadera discusión estética, si hay una idea de belleza asociada a la sostenibilidad, ésta podrá interesar a la arquitectura de una forma no circunstancial y tendrá sentido trabajar sobre ella". En su "Atlas Pintoresco", Ábalos apunta una serie de estrategias a explorar trazando una narrativa sobre los parques y los paisajes artificiales, sobre la observación, la cartografía y el escrutinio de aquello que no se conoce y su transformación en imágenes ${ }^{4}$.

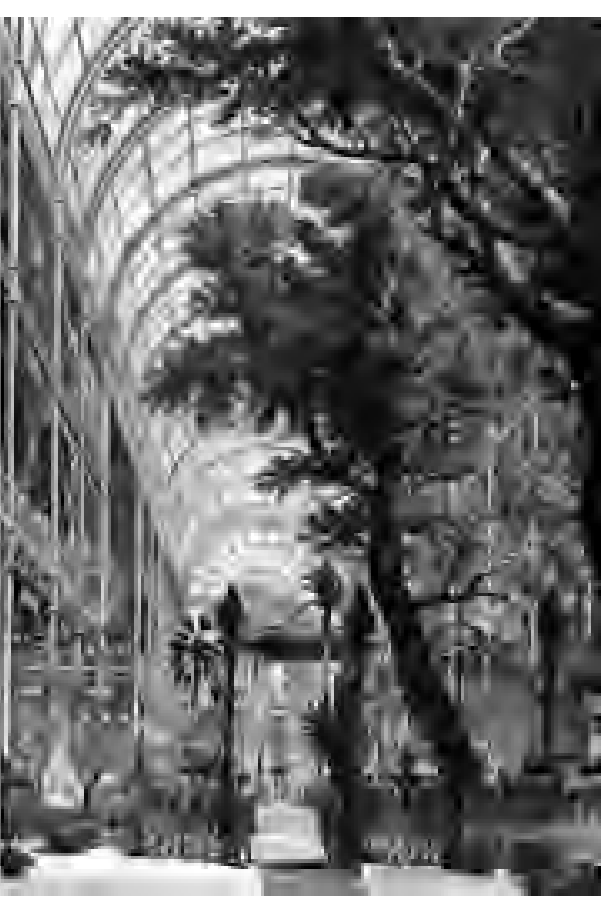

Fig. 02

A) Villle Radieuse, Le Corbusier, 1922. B) Crystal Palace, Joseph Paxton, Gran Exposición de Londres de 1951. 
Fig. 03

Maqueta, Mediateca de Sendai, Toyo Ito, 1995-2001.

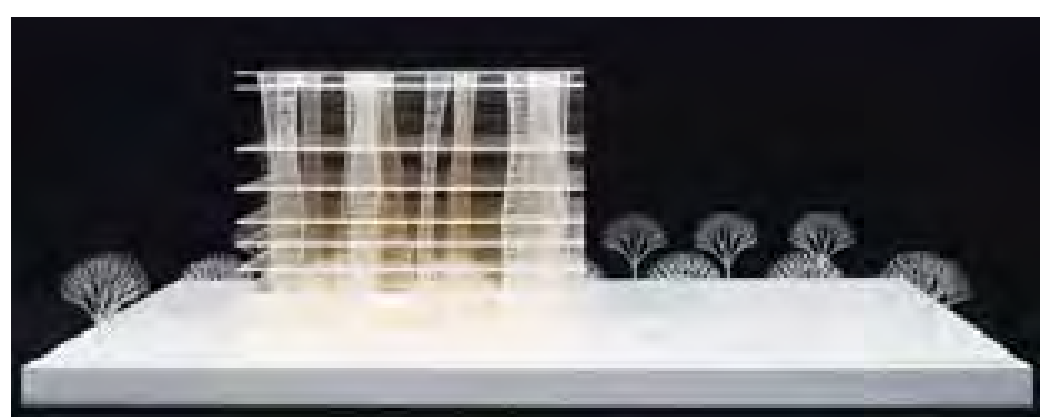

03

La idea de 'naturalización' debe situarse en este contexto y entenderse como superación de prácticas autoproclamadas sostenibles o naturalistas. Estas últimas parecen haberse polarizado en dos estrategias opuestas: una asociada a lo puramente técnico y otra a una especie de expresionismo de la estética natural. En el primer caso, la arquitectura parece reducirse a mera estadística, justificando cualquier solución desde el cumplimiento de los coeficientes de consumo cero y la obtención del certificado de 'edificio verde'. Mientras que en el segundo caso, por lo general, encontramos una traslación literal de las formas naturales, bien a través de una fachada vegetal que envuelve el edificio o convirtiendo el edificio directamente en paisaje. Frente a este marco dominado esencialmente por la mercadotecnia que ha hecho de la sostenibilidad su palabra estrella y repleto de actitudes de poco alcance, las siguientes propuestas aportan temas sobre el modo de visualizar la naturaleza construyendo una relación crítica y reflexiva con la arquitectura.

NATURALEZA

COMO METÁFORA:

ARQUITECTÓNICO

MODERNO.

MEDIATECA DE SENDAI,

TOYO ITO, 1995-200O
RENOVACIÓN DEL ESPACIO

\author{
"Con su sencilla construcción, la mediateca tiene vocación de \\ convertirse en el arquetipo de una nueva arquitectura comple- \\ tamente nueva. Servirá de lugar de encuentro de los dos \\ cuerpos del ser humano contemporáneo, el cuerpo que \\ contiene el flujo de electrones y el cuerpo primitivo sensible a \\ la naturaleza"5.
}

La Mediateca de Sendai es fruto de un concurso internacional que llevó a Toyo Ito a realizar, durante los años 1995 y 2001, uno de sus edificios más celebrados. Su imagen icónica, la innovación estructural o la flexibilidad programática otorgan al edificio un indudable interés, pero cabe añadir el intento por representar algunos temas de la cultura contemporánea como la condición de fluidez, la multiplicidad funcional, la virtualidad de los límites o los avances de la tecnología digital y los medios electrónicos. Estos temas también han sido desarrollados por Ito en diversos textos en un esfuerzo por profundizar en los cambios de la sociedad contemporánea.

A pesar de ser consciente de las especificidad de la arquitectura reconociendo sus códigos y una determinada tradición, Ito considera imprescindible encontrar en estos temas parte del significado de la arquitectura. Con el objetivo de introducir determinados significados en su obra resulta frecuente el uso que hace de la metáfora, como se ejemplifica en la Mediateca, encontrando en la interpretación de la naturaleza o las formas naturales su principal foco de atención. Cabe señalar que metáfora procede del término griego 'mataphora', que viene a decir 
'traslación' o 'transferencia', y que se basa en la articulación de relaciones de una cosa con algo distinto de ella misma para definir un campo de reconocimientos y asociación al tiempo que transmisión de significado6.

La idea de columna como árbol domina la concepción de la Mediateca; mientras que la definición espacial del edificio parece asentarse en la idea de bosque, con zonas que oscilan entre lo abierto y lo cerrado o lo luminoso y lo obscuro, para una composición de ambientes variados (fig. 03). Pero también se recurre a los fenómenos atmosféricos con la intención de sugerir un ambiente compuesto por actividades fluctuantes; en este caso los flujos de información sustituyen a los naturales. Las diversas actividades de las plantas se conciben desde este intercambio de información a través de sistemas convencionales o micro-electrónicos. La multiplicidad de capas (programas) o el fenomenalismo completan esta asociación, validando la idea de que "la arquitectura no debe reclamar su propia forma física sino que debe convertirse en un dispositivo para interpretar la forma como fenómeno"7.

En el texto "Arquitectura pública como punto de paso" Ito aboga por una ciudad compuesta por edificios públicos transparentes y permeables, a modo de "objetos relativizados", con funciones y espacios heterogéneos que fomenten el intercambio de flujos (fig. 04). Siguiendo este principio, la Mediateca se asienta en un sistema abierto de actividades intercambiables para tratar de lograr cierta indefinición funcional. La integración de una galería de arte, una biblioteca municipal y un centro de medios audiovisuales, parece basarse en un proceso orgánico de descomposición, combinación o fusión.

En la descripción que realiza Ito de la Mediateca, a modo de arquetipo, descompone la forma del edificio en tres categorías: placas (planchas de acero), tubos (estructura de acero) y piel (fachada de vidrio). Se recurre, nuevamente, al ámbito de las formas naturales. Las placas se traducen en losas cuadradas de acero de 50 metros de lado (siete en total), representadas en los dibujos como lugares que contienen

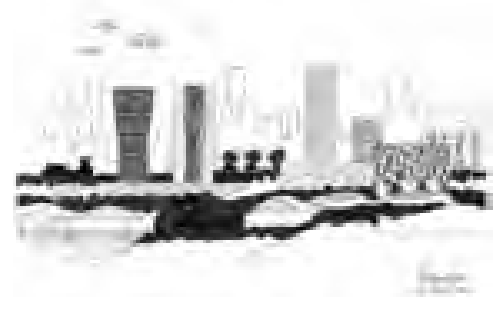

04

Fig. 04

Idea de ciudad; donde medio natural $y$ construido forman una unidad indivisible que favorece la fluidez y la intercambiabilidad. Croquis, Toyo Ito, 19 febrero 2011.

Fig. 05

Croquis de las plantas y la sección, Mediateca de Sendai, Toyo Ito, 1995-2001.

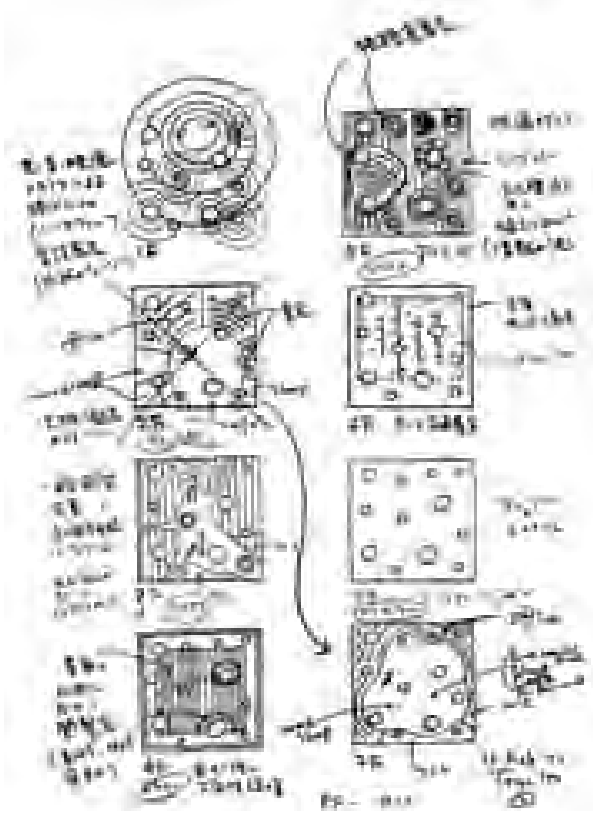

05

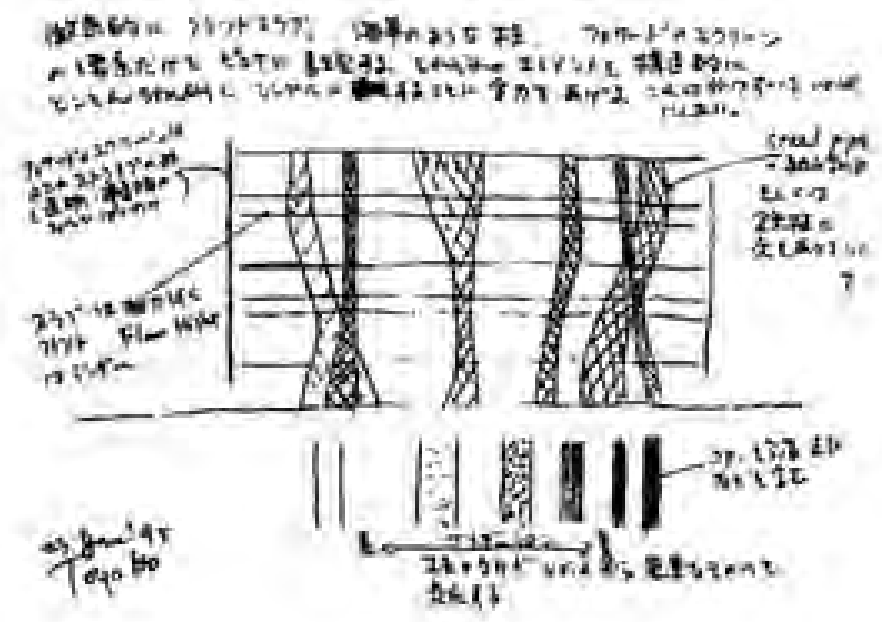


Fig. 06

Planta, Stortoget, Kalmar, Caruso St. John, 1999-2003.
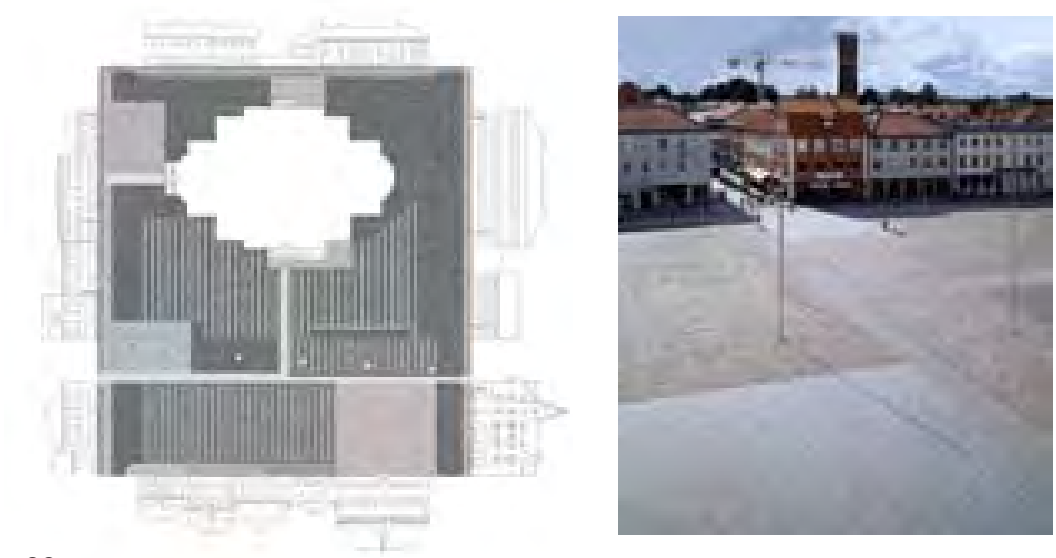

06

el flujo de los usuarios y de los objetos. Se favorece con ello un sistema de densidades variables con la convicción de garantizar una alta diversidad (la iluminación artificial también adquiere un papel relevante en el intercambio y las variaciones de la planta) ${ }^{8}$. La sección también recoge esta libertad planimétrica a través de la variación de alturas entre placas que acentúa la flexibilidad del sistema. Cabe añadir que a la libertad que encontramos en el 'sistema dominó' de Le Corbusier o en el 'espacio universal' de Mies, en este caso se introduce cierto carácter azaroso (fig. 05)'.

La estructura de la Mediateca sirve para ordenar y cualificar la planta, ya que su posición estratégica en cada plataforma garantiza la ocupación y una adecuada distribución del espacio. Se compone de 13 tubos de formas biomórficas que, como si de árboles se trataran, penetran verticalmente en las placas y las sostienen. Éstos, además del transporte de cargas, permiten el flujo de diversas energías, como la luz, el aire, el agua o el sonido. La concepción de la estructura como algo más que sistema portante se observa también en su dimensión ornamental, superando las convenciones que insisten en la independencia de ambas. La función estructural, así, pierde su cualidad de fundamento para camuflarse en un sistema decorativo que define ambientes y acentúa las ocupaciones.

En cuanto a la corporeidad de la arquitectura, en la Mediateca surge la tensión entre los requisitos materiales de cobijo y el deseo de diluir el recinto y los límites. La metáfora de la piel muestra el deseo de lto de convertir la fachada en un interfaz que pudiera articular lo tangible y lo intangible o lo natural y lo artificial. La imposibilidad real de este hecho, ya que toda forma arquitectónica u objeto material siempre se sustenta en una determinada expresión tectónica, revela con mayor claridad las limitaciones de tales tentativas. Ito, sin embargo, parece deleitarse en esta ambigüedad, de ahí que su búsqueda de lo virtual se desarrolle en torno a las preocupaciones por la tectónica y su empleo con fines metafóricos.

El espacio neutro y abstracto elegido como punto de partida para la Mediateca, aludiendo a la tradición moderna, sufre un proceso de relleno a través de la recuperación de la cualidad figurativa de la arquitectura y de la ornamentación literal y metafórica. Tal vez, porque la paradoja se asume como forma de pensamiento, pero también porque el rechazo de la forma no está reñido con las técnicas del contraste entre fondo y figura. Este hecho ejemplifica, de un modo elocuente, 
el recurso de la metáfora por parte de Toyo Ito, acentuando que la idea de naturaleza no deja de ser un constructo cultural. En este sentido, esta obra deja entrever que lo que más le interesa al arquitecto japonés no es su contemplación ni sus valores higienistas (los modelos romántico y moderno) sino su capacidad para mostrarnos, para darnos claves con las que interpretar los cambios culturales que la era digital ha introducido en nuestras vidas.

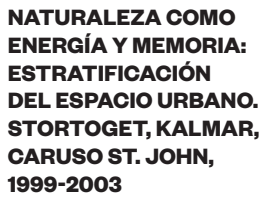

"En mi primera visita a la zona, incluso con ochenta automóviles estacionados en el lugar, me conmovió la belleza de la superficie de piedra, la variedad de tamaños y colores del material y el modo en que brillaban al sol. La historia física de Stortoget se basa en el movimiento de estas piedras autóctonas desde las tierras agrícolas vecinas, donde se encontraban apiladas formando lindes, para utilizarlas en las calles y la plaza de la localidad barroca. La presencia de esta piedra en el centro de Kalmar recuerda su transformación física, casi primitiva, de zona rural a lugar urbano"10.

El proyecto de Caruso St John, en colaboración con la artista sueca Eva Löfdahl, aborda la tarea de renovar la plaza principal de Kalmar con la finalidad de recuperar la identidad perdida tras el paso del tiempo y la fractura provocada por el tráfico rodado. Frente a la restitución de la unidad haciendo tabula rasa, los autores conservan la fragmentación existente restituyendo los patrones que a lo largo del tiempo habían caracterizado el plano del suelo. Esta estrategia pretende superar la condición artificiosa que denotan muchas intervenciones contemporáneas, asumiendo los cambios surgidos en el tiempo en la evolución natural del propio espacio urbano.

Caruso St John plantean una solución que constituye dos sistemas de orden. En primer lugar, un sistema regulador basado en trazados perpendiculares que articula los flujos y los movimientos (construidos a base de texturas más lisas como adoquines de granito y losas prefabricadas de hormigón); y, en segundo lugar, la definición específica de los ámbitos definidos por el primer sistema a través de cambios sutiles en los despieces y los colores de las piedras de granito (cantos rodados de granito de distinta dimensión utilizados originalmente en la plaza). Si el primero otorga unidad a toda la extensión de la plaza, el segundo posibilita una riqueza expresiva en la textura del suelo que remite a la condición orgánica y heterogénea de las formas naturales (fig. 06).

La estrategia de intervención va más allá del ámbito de la propia plaza. La definición del sistema de trazados, remite tanto a su perímetro como al entorno más lejano, para hacer partícipe al propio espacio de una red de flujos energéticos, culturales y materiales que invoca la memoria de toda la ciudad. Esta definición de lugar, que trata de ampliar su compresión como mera interpretación tipológica de los trazados, disuelve la permanencia de las trazas en una relación múltiple entre el espacio renovado y aquello que lo rodea y lo contiene.

La intervención también busca la abstracción del propio plano del suelo, sin acotar ni definir ámbitos para usos específicos. Esta polivalencia, parece asumir la memoria de un espacio que ha sido objeto de múltiples usos a lo largo de sus 300 años, que oscilan desde las representaciones políticas, militares o religiosas hasta servir como foco comercial de la ciudad. Se evita cualquier adorno o mobiliario pintoresco, para 

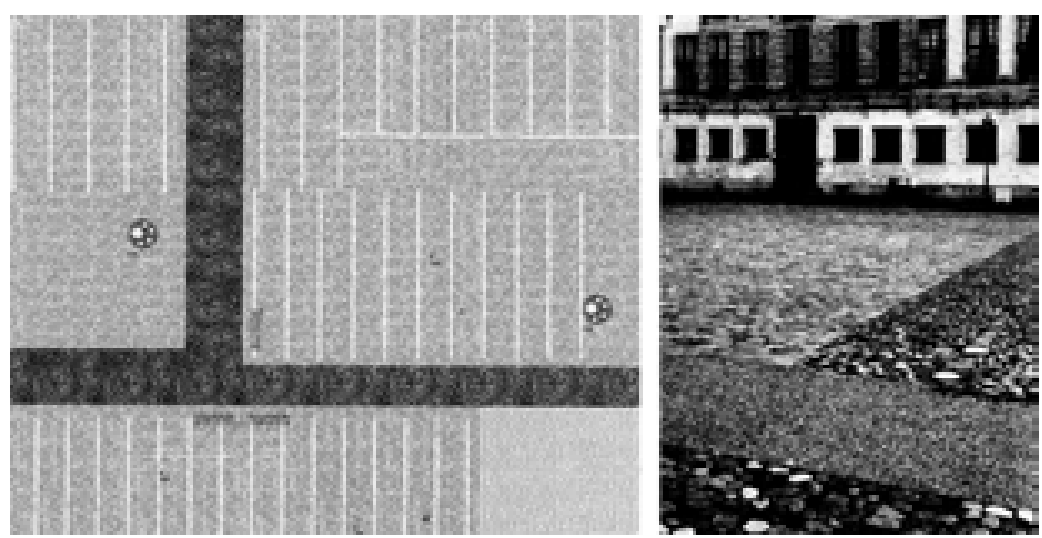

07
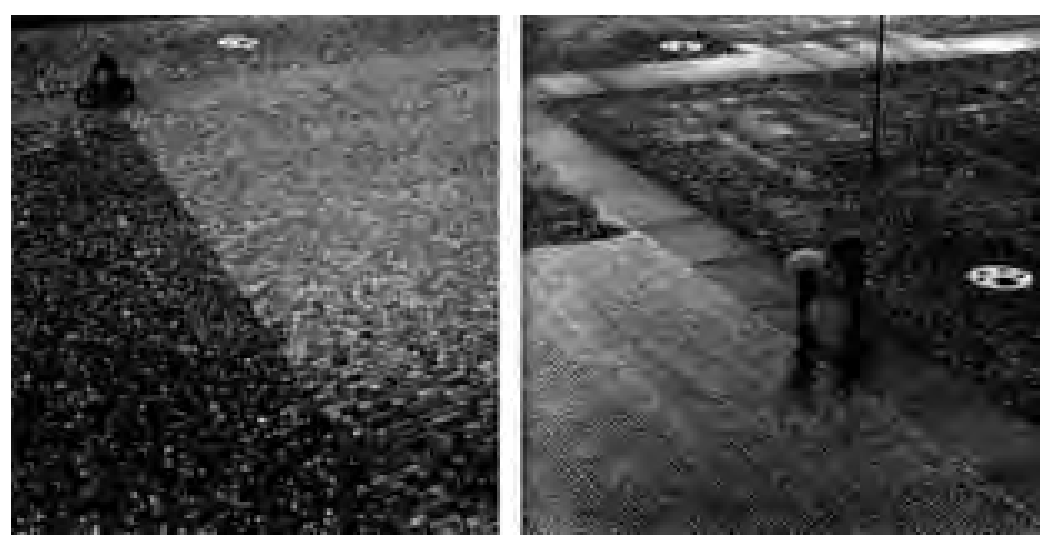

08

acentuar la propia textura del plano del suelo. En este sentido, el pavimento adquiere una clara presencia física, a modo de superficie orgánica, gracias al espesor y al claroscuro de los diversos patrones de la piedra (fig. 07).

La materia se convierte en objeto activo y sensible, en depósito de energía con el que operar. Los arquitectos prestan atención al potencial configurador de este depósito conservado en los desplazamientos y recolocaciones de los adoquines y los cantos rodados. La organización de la plaza, parece encontrar en esta interpretación de la materia la identidad de lugar, de un genius loci renovado. La posibilidad de comprender la plaza desde su memoria material supone una mirada reflexiva sobre el medio y de los entornos naturales y urbanos. Los restos pétreos desplazados y reunidos como si de procesos entrópicos naturales se tratara, convierten a Stortorget en signo tangible de la propia vida de la ciudad. Como señala Adam Caruso: "Podemos imaginar el medio como algo que puede abarcar tanto el esfuerzo humano como la materia, un territorio en el que se pueden establecer conexiones entre la energía y la cultura"".

En Stortoget, la idea de espacio urbano viene enriquecida

Fig. 07

Detalle del pavimento, Stortoget, Kalmar, Caruso St. John, 1999-2003.

Fig. 08

Stortoget, Kalmar, Caruso St. John, 19992003. a través de estas conexiones entre materia y energía, a modo de estratificación de significados que busca, en todo caso, una arquitectura que pudiera adoptar un ámbito medioambiental más amplio. El transporte de piedras de otros lugares de la ciudad para reubicarlas dentro del nuevo orden se concibe como operación donde los flujos de energía adquieren su expresión y se acumulan en un material que los hace sensibles (fig. 08). 
Esto supone un intercambio de energía que enmarca una mirada sobre la forma, la materia, la memoria o el tiempo dentro de un proceso que trata de establecer un vínculo homólogo a los del medio natural.

Las piedras de Kalmar, por lo tanto, se muestran transportadoras de energía y memoria; de un pasado que se presenta en la superficie del suelo de la plaza a través de capas de materia. Todo ello dentro de un proceso constituido por estratos cuyo significado se recoge en los mismos cantos rodados. El tiempo se interpreta como una variable discontinua y no lineal, liberada de ataduras para conectar la experiencia de la arquitectura al lugar y a la vida. La plaza, asimismo, se revela como resultado de flujos de energía que reconocen un sedimento del pasado; suma de múltiples niveles que fija una instantánea con la que pasado y futuro se reducen a un presente concreto. Bien podríamos considerar la plaza de Kalmar como ese lugar instantáneo, cuyas piedras de granito portadoras de energía y de memoria se disuelven entre destellos fugaces y ruidos de agua.

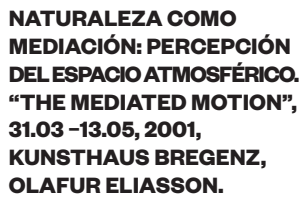

"Al integrar al espectador, o más bien, al acto mismo de mirar como parte de la tarea del museo, el interés se ha trasladado de la cosa experimentada a la experiencia en sí. Ponemos en escena los artefactos, pero lo que es más importante, ponemos en escena el modo en cómo se perciben los artefactos. Cultivamos la naturaleza en los paisajes (la imagen

extraída de mirar la naturaleza). Así, para eludir la insistencia del museo en la existencia de una naturaleza (si se busca con ahínco), resulta crucial no solo reconocer que la experiencia misma forma parte del proceso, sino, lo que es más importante, que la experiencia debe presentarse manifiestamente al espectador"12.

La instalación "The Mediated Motion" plantea la transformación física del espacio arquitectónico de la Kunsthaus de Bregenz a través de la inserción de distintos estratos de naturaleza que el espectador contempla a lo largo de sucesivas plantas. La estricta geometría del edificio de Peter Zumthor se convierte en una especie de naturaleza miniaturizada pero sublime y el museo en una secuencia de espacios atmosféricos que envuelve a los visitantes con su densidad estética. La eficacia artística de la instalación estriba en el inmediato shock perceptivo producido por la atmósfera de cada paisaje construido, que lejos de descansar en una ilusión efectista, subraya una rigurosa puesta en escena. La relación entre edificio y naturaleza, también artificial, constituye un dialogo donde lo natural parece emanar de lo artificial y lo artificial de lo natural.

La obra expuesta se convierte en contexto y la naturaleza en paisaje. Eliasson cuenta con la colaboración del arquitecto paisajista Günther Vogt, con la idea de definir un recorrido, ligado al movimiento en espiral en el que se basa el edificio de Zumthor, donde el espectador se desplaza de una situación paisajística a otra. Madera con setas, un pontón de madera sobre aguas cubiertas de lentejas acuáticas, un plano de tierra contaminada y, finalmente, una sala llena de niebla con un puente colgante. La instalación se debate en una tensión entre el arte conceptual, cerebral, de raíz científica, y un arte emocional, subjetivo, basado en la experiencia.

El título de la exposición hace referencia a dos aspectos que el artista danés considera esenciales en la construcción de relaciones entre el ser humano y el medio: la mediación y el movimiento. Para 
Eliasson la mediación supone "el grado de representación en la experiencia de una situación"; grados o niveles en continuo estado de flujo al variar según los factores que intervienen en cada caso. En este sentido, la mediación se plantea como vía que posibilita la evaluación, la crítica y la reflexión y por lo tanto, uno de los valores centrales de la práctica artística y cultural. En cuanto al movimiento, la exposición pone en valor la idea de recorrido y flujo, a través del cual el espectador va construyendo una actitud crítica hacia lo que observa y percibe. El recorrido por las salas de la exposición posee algo de atracción de feria. El visitante permanece a la expectativa de la sorpresa que le depara la siguiente sala, aguarda el siguiente truco (fig. 09). Como señala Eliasson: "en los cuatro niveles, el olor, la niebla, el agua, las plantas o el cultivo, producen que la estricta arquitectura ortogonal de vidrio y hormigón de Peter Zumthor se metamorfosee en un campo de experiencias y de conciencia de estas experiencias"13.

La instalación también pretende hacernos reflexionar sobre el tiempo atmosférico y su capacidad de mediación, entendido como "naturaleza en la ciudad" cuyo interés reside en su imprevisibilidad y la capacidad de cambio. En la exposición, Eliasson manipula algunos elementos básicos del tiempo atmosférico como el agua, la luz, la temperatura o la misma presión atmosférica. Mediante la introducción del fenómeno natural, induce al observador a reflexionar sobre cómo percibimos el mundo natural y cómo éste afecta a nuestra percepción del entorno; "como habitantes, hemos crecido habituados al clima mediado por la ciudad a través de nuestra experiencia progresiva del espacio urbano"14.

El uso de la naturaleza, también viene acompañado de un proceso de desmaterialización, donde las atmósferas generadas parecen diluir los propios límites que las contienen. Este hecho, acentúa la condición de modelo atmosférico en la que pretende convertirse la exposición y hace reflexionar sobre las propiedades cambiantes del espacio arquitectónico. Eliasson se vale de la luz, de proyecciones o de la creación de niebla para manipular o anular los puntos de referencia del espectador y provocar sensaciones que en algunos casos podrían calificarse como oníricas. Todo ello amplía los mecanismos perceptivos más allá de los meramente visuales, donde lo visible incluye lo no visible. Bajo esta óptica, Eliasson apunta a una realidad arquitectónica donde tenga cabida una reflexión más profunda sobre el espacio atmosférico. Estos temas tendrán continuidad dos años más tarde en la instalación "The Weather Project" en la sala de turbinas de la Tate Modern de Londres (fig. 10) ${ }^{15}$.

Ambas instalaciones ponen de relieve la idea de paisaje como imagen proyectada de la naturaleza. Tanto en la Kunsthaus como en la Tate Modern, la relación entre naturaleza y arquitectura, debe entenderse desde la idea de mediación, donde el sujeto se convierte en protagonista en su interacción con la obra artística. El artista danés también pretende dejar atrás el debate entre 'naturaleza versus cultura', para plantear una reflexión que inste al espectador a reconsiderar sus experiencias en un campo constituido por ambas. Eliasson propone que lo natural no se entienda como despensa de formas alternativas a las convencionales, sino como foco de energía para que nuestras vidas se activen y como telón de fondo donde se proyecten. RA 
Fig. 09

Movimiento y mediación. "The Mediated Motion", 31.03-13.05 2001, Kunsthaus Bregenz, Olafur Eliasson.

Fig. 10

A) “The Mediated Motion", 31.03-13.05, 2001, Kunsthaus Bregenz.

B) “The Weather Project", 16.10.2003 21.03.2004, Tate Modern London. Olafur Eliasson.
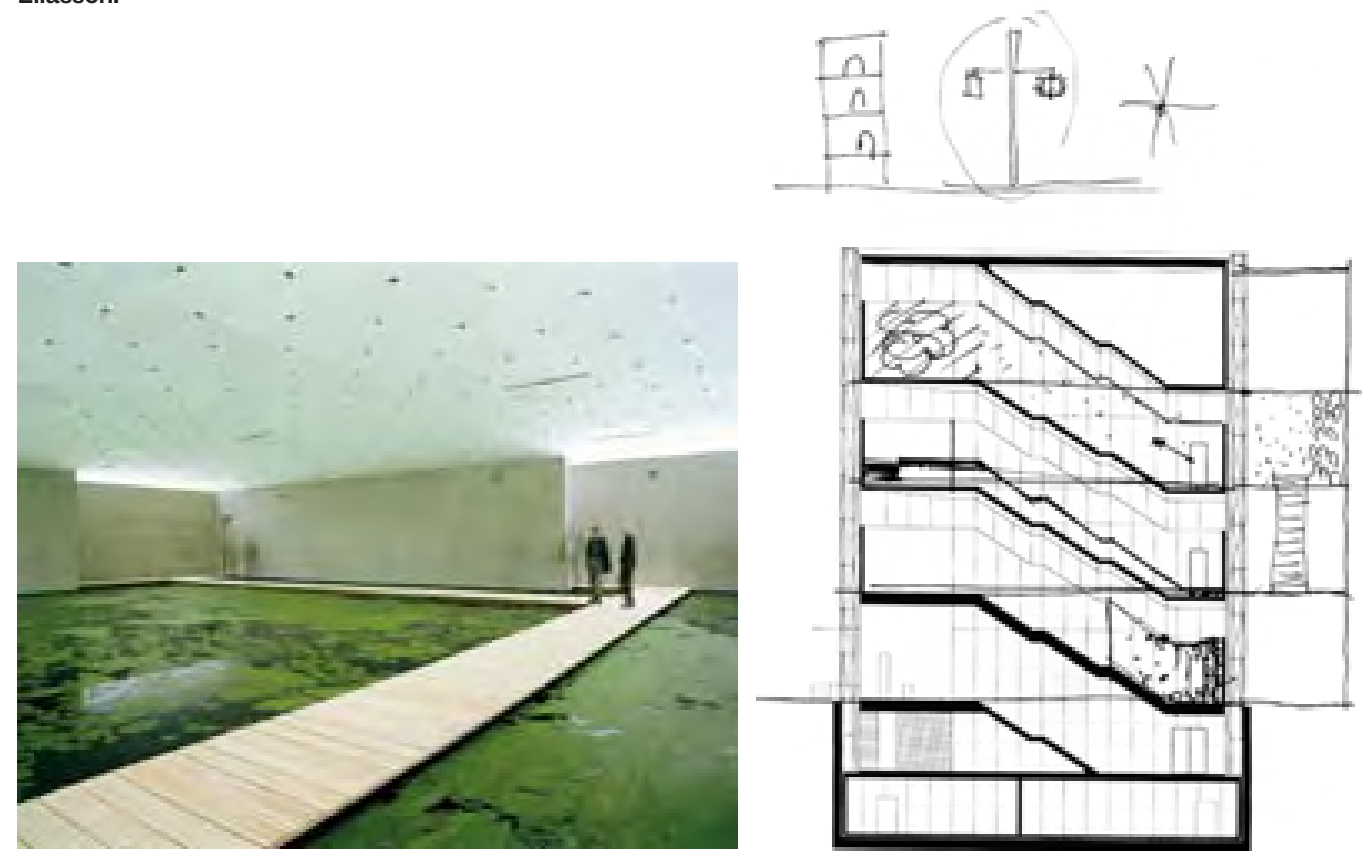

09
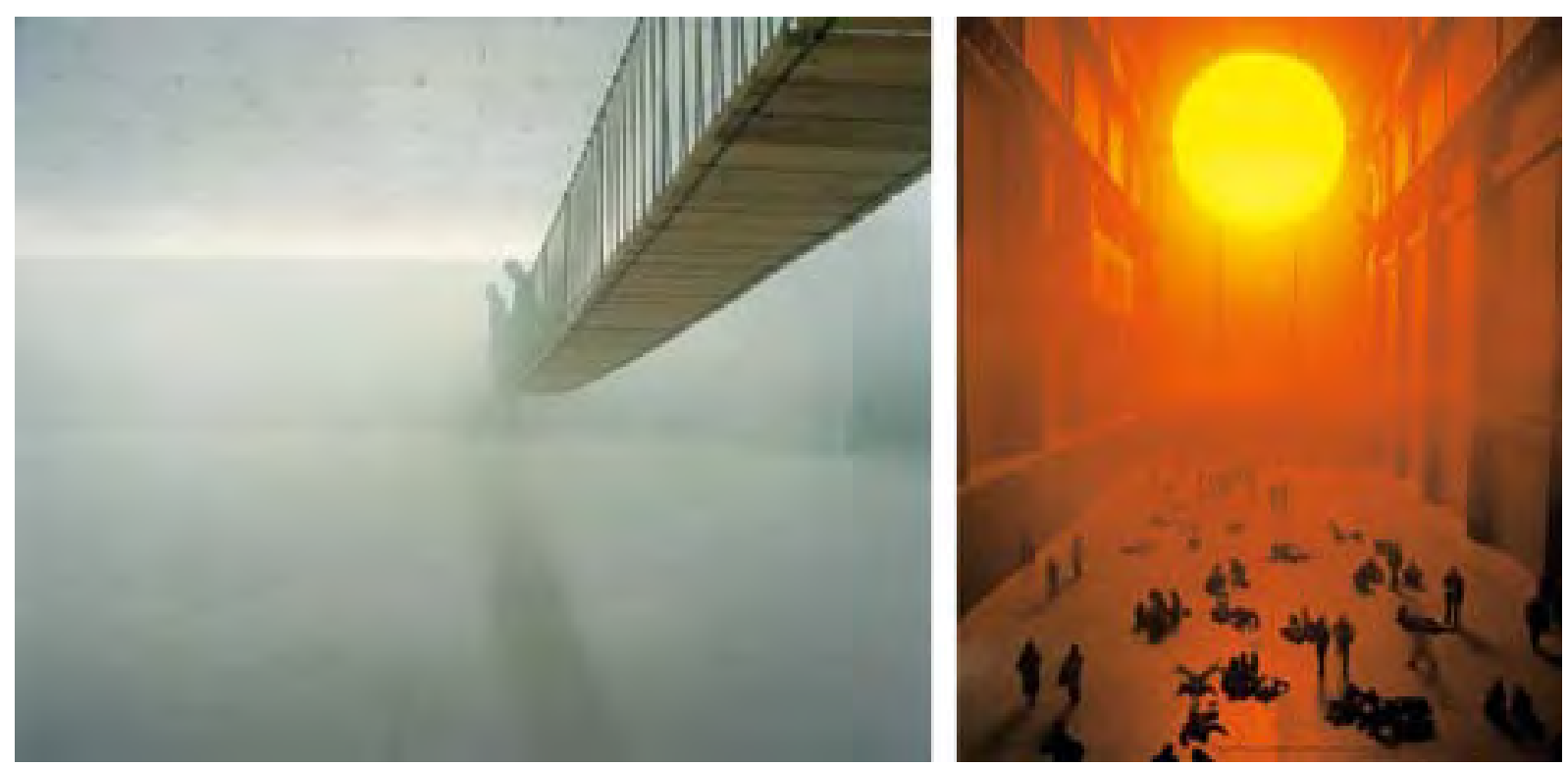
01. ELIASSON, Olafur, Leer es respirar, es devenir, Gustavo Gili, Barcelona, 2012, p. 24.

02. SMITHSON, Robert, "A Museum of Language in the Vicinity of Art", Art International, marzo 1968. También en FLAM, Jack. Robert Smithson: The Collected Writings, University of California Press, Berkeley, Los Ángeles, Londres, 1996.

03. URSPRUNG, Philip, Brechas y conexiones: ensayos sobre arquitectura, arte y economía, Puente Editores, Barcelona, 2016.

04. El "Atlas Pintoresco" constituye un recorrido que conduce al lector a través de la historia de la invención de la naturaleza en los siglos XIX y XX, en sintonía con las ideas de Robert Smithson. ÁBALOS, Iñaki, Atlas pintoresco. Vol. 1: el observatorio pintoresco. Vol. 2: los viajes, Gustavo Gili, Barcelona, 2005 y 2008. Consultar también, ÁBALOS, Iñaki, "La belleza termodinámica", en "Ábalos+Sentkiewicz", 2G, 2010, n. 56, pp. 127-136.

05. ITO, Toyo, "Mediateca en Sendai", en “Toyo Ito, Sección 1997", 2G, 1997, n. 2, p. 26.

06. Como señala el filósofo Mark Johnson la metáfora debe entenderse como "un penetrante modo de comprensión a través del cual trasladamos modelos de un cierto campo de nuestra experiencia a fin de estructurar otro campo de diferente tipo". JOHNSON, Mark, The body in the mind: the bodily basis of meaning, imagination, and reason, University of Chicago Press, Chicago, 1987, p. XIII.

07. ITO, Toyo. "Un jardín de microchips. La imagen de la arquitectura en la era microelectrónica". En Escritos, Colegio Oficial de Aparejadores y Arquitectos Técnicos de Murcia, Murcia, 2000, pp. 146-147.

08. En cuanto a la iluminación artificial, se hace claramente visible en las imágenes del edificio. Las diferentes alturas de cada planta adquieren presencia y relevancia formal y espacial como consecuencia de las variaciones en la iluminación; diferencia en la intensidad, en el color o en la calidez. Estas variaciones son las encargadas de codificar las diversidades espaciales entre las plantas, lo cual constituye una utilización claramente anti-moderna de la tecnología, ya que su representación mediante una determinada iconografía se sustituye por la manipulación espacial de la misma.
09. "Se dice que en la arquitectura moderna ha habido dos propuestas de un sistema de arquitectura entendida como 'prototipo'. Una es el 'espacio universal' de Mies van der Rohe, y la otra el sistema 'do-mino' de Le Corbusier. El primero intenta componer el espacio dividiéndolo en cuadrículas uniformes tridimensionales... Continuando la retícula, teóricamente se puede prolongar el espacio hasta el infinito, tanto horizontal como verticalmente. El sistema 'Do-mino' se compone de forjados planos y de columnas sin vigas. Podemos decir que el sistema estructural de Sendai está próximo al sistema 'Do-mino"'. ITO, Toyo, Escritos. Colegio Oficial de Aparejadores y Arquitectos Técnicos de Murcia, Murcia, 2000.

10. CARUSO, Adam, The feeling of things: escritos de arquitectura, Polígrafa, Barcelona, 2008, pp. 19-20.

\section{Ibid. p. 15.}

12. ELIASSON, Olafur. Leer es respirar, es devenir, op. cit., pp. 24-25.

\section{3. "En mi búsqueda de pistas para} desentrañar el edificio -puesto que sin duda, el edificio está lleno de ideas preconcebidas acerca de cómo ver el edificio y experimentar con él-, descubrí la generosidad del movimiento en espiral que te lleva se una planta a la siguiente. Al intentar sacar el máximo partido a vuestra mirada instruida e integraros como principales intérprete de la exposición, me di cuenta de que la clave estaba en aumentar el principio del movimiento. Puesto que vuestro movimiento y vuestra orientación forman un proceso en el tiempo, busqué un oportunidad y un medio para convertir este proceso concreto en objeto. Hay un campo en particular en el que se ha cultivado el proceso como objeto, la arquitectura del paisaje, de ahí que me haya fijado en Güenther Vogt, cuyas ideas acerca de los procesos de cultivo has sido fuente clarividente para el desarrollo de este proyecto". Ibid. p. 14.

14. Señala Eliasson que "uno podría equivocadamente pasar por alto una situación como un estado "natural" de cosas, y no ser consciente de las construcciones que subyacen bajo esa situación. El desafío de orientarnos en un terreno mediado es, pues, ver a través y saber cuándo, en qué grado y mediante quién una situación ha sido mediada; ser conscientes de la relación de una situación con el tiempo. Cualquier museo debe permitir que el visitante entienda que la ideología institucional y la exposición son en sí mismas una construcción y no un estado de "verdad" más elevado. Cuando podemos "ver a través" de la mediación de una situación, cuando es transparente, podemos experimentar un grado de autoconsciencia más intenso debido al potencial auto-evaluador que existe dentro de una situación como esta". lbid.

\section{En esta instalación "The Wather} Project", la representación del sol y el cielo domina el espacio de la Sala de turbinas de la Tate Modern de Londres. Una fina neblina se expande por el espacio, como si se hubiera introducido desde el exterior. A lo largo del día, la niebla varía simulando los fenómenos atmosféricos. Mirando hacia el techo para contemplar como desaparece la niebla, vemos que el techo de la sala de turbinas ha desaparecido, reemplazado por el reflejo del suelo. Al fondo de la sala, encontramos un círculo gigante formado por cientos de lámparas de mono-frecuencia. El arco repetido en el espejo produce una esfera de brillante radicación ligando el espacio real con la mismo reflexión. Usado generalmente en el alumbrado público, las lámparas de mono-frecuencia emiten una luz de mínima frecuencia que mitiga los colores que no sean el amarillo o el negro. Todo ello transforma el campo vidual alrededor del sol en un vasto paisaje duotono. Consultar: PRIETO, Eduardo, "Ars meteorológica. Naturaleza y arquitectura en la obra de Olafur Eliasson", en Arquitectura Viva, 2011, n. 141, pp. $74-75$. 


\section{Referencias bibliográficas}

-ÁBALOS, Iñaki; SENTKIEWICZ, Renata; ORTEGA, Lluis. Ensayos sobre termodinámica, arquitectura y belleza: Ábalos Sentkiewicz. Actar, Nueva York, 2015.

- BUDD, Malcolm. The Aesthetic Appreciation of Nature: Essays on the Aesthetics of Nature. Oxford University Press, New York, 2002.

- CARUSO, Adam. The feeling of things: escritos de arquitectura. Polígrafa, Barcelona, 2008.

- CARUSO, Adam; ST JOHN, Peter. Caruso St John, 1993-2013: forma y resistencia $=$ form and resistance. El Croquis, 166, El Escorial (Madrid), 2013.

- ELIASSON, Olafur. Leer es respirar, es devenir. Gustavo Gili, Barcelona, 2012.

- ELIASSON, Olafur. Studio Olafur Eliasson An encyclopedia. Taschen, Köln, 2008.

- FLAM, Jack. Robert Smithson: The Collected Writings. University of California Press, Berkeley, Los Ángeles, Londres, 1996.

- ITO, Toyo. Escritos. Colegio Oficial de Aparejadores y Arquitectos Técnicos de Murcia, Murcia, 2000.

- ITO, Toyo. Toyo Ito 2001-2005. El Croquis, 123, El Escorial (Madrid), 2004.

- LEE, Sang. Aesthetics of sustainable architecture. 010 Publishers, Rotterdam, 2011.

- URSPRUNG, Philip. Brechas y conexiones: ensayos sobre arquitectura, arte $y$ economía. Puente Editores, Barcelona, 2016.

- ZUMTHOR, Peter. Peter Zumthor atmósferas: Entornos arquitectónicos - las cosas a mi alrededor. Gustavo Gili, Barcelona, 2006. 
28. "Declaration of the Vancouver Symposium", en Ekistics, 42, 252, 1976, pp. 267-272.

\section{ANKER, Peder, "The} Closed World of Ecological Architecture", en Journal of Architecture, 10:5, 2005, pp. 527-552.

30. McHARG, Ian, Design With Nature, Garden City, Nueva York, Doubleday, 1969. Traducido al español como Proyectar con la naturaleza, Barcelona, Gustavo Gili, 2000.

31. McHARG, lan, "Architecture in an Ecological View of the World", en AIA Journal, 54, noviembre de 1970, pp. 47-51.

32. Citado en GREEN, op. cit.

33. "Executive Summary, Observations for the Future", op. cit., p. 7.

34. Véase, por ejemplo, BURANYI, Stephen, "Rise of the racist robots - how $\mathrm{Al}$ is learning all our worst impulses", en The Guardian, 8 de agosto de 2017, <https://www.theguardian.com/inequality/2017/ aug/08/rise-of-the-racistrobots-how-ai-is-learning-allour-worst-impulses>, último acceso el 18 de febrero de 2018.

35. JOPPA, op. cit.

\section{Imágenes}

01. Jardín de la Fundación Ford, Nueva York, del arquitecto paisajista Dan Kiley, 1964. Fotografía de David Leventi, 2013. Cortesía de Rick Wester Fine Art, New York.

02. "Cuadro de mandos medioambiental", diseño de interfaz de $\mathrm{N}$ sketch Inc. (Yuki Anezaki y Kohei Tsuji) para Fujitsu. Participante de los premios iF Design Awards 2015.

03. Jardín de la Fundación Ford, Nueva York, del arquitecto paisajista Dan Kiley, 1964. Fotografía de David Leventi, 2013. Cortesía de Rick Wester Fine Art, New York.

04. El Tele-Garden de Ken Goldberg y Joseph Santarromana, University of Southern California y Ars Electronica Museum, Linz, Austria, 19952004.

\section{The Naturalisation of Architectural Space. Three Critical Positions far from Naturalism Javier de Esteban}

The article analyses three coincident projects, developed at the turn of the century, which delve into the relation between nature and architectural space. In the three cases, the interpretation of nature, understood as a cultural construct, is integrated into the architectural conception and the ideation itself. Under what could be called a 'naturalisation' process, these practices get away from those self-proclaimed sustainable or naturalist, usually of short-term view, recovering some traditions and sharing a critical attitude that allow us to reflect on new ways of visualizing nature and its relation with architecture.

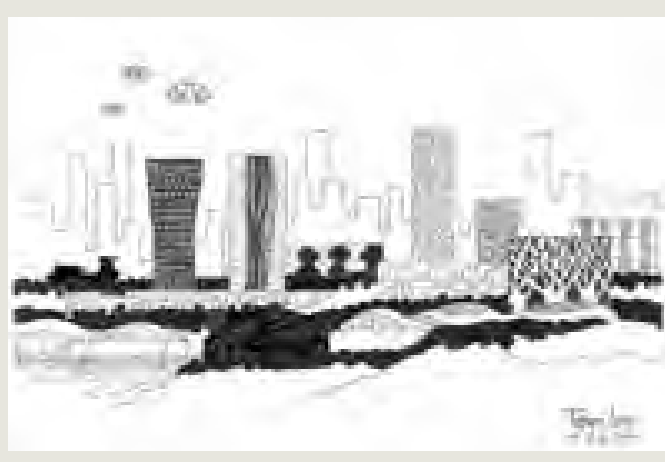

NATURALISATION VERSUS NATURALISM
"So what is it that I know? Is it nature? Nature as such has no 'real' essence - no truthful secrets to be revealed. I have not come closer to anything essential other than myself and, besides, isn't nature a cultural state anyway? What I have come to know better is my own relation to so-called nature (i.e., my capacity to orient myself in this particular space), my ability to see and sense and move through the landscapes around me. Looking at nature, I find nothing... only my own relationship to the spaces, or aspects of my relationship to them. We see nature with cultivated eyes. Again, there is no truthful nature; there is only your and my construct of such. Just by looking at nature, we cultivate it into an image"1.

The American artist Robert Smithson described nature as "simply another fiction of the eighteenth and nineteenth centuries". This provocative statement come to say that nature should be understood as a cultural construct that has been different interpretations by human being throughout the stages of history. As can be interpreted in works such as the iconic Spiral Jetty, Smithson tries to leave behind numerous conventions to delve into the relationship between technology and natural forms. The artist emphasizes that nature can not be separated from its own interpretation, showing an interesting framework based on technological changes and their possibilities in its visualization and understanding ${ }^{2}$.

This conception acquires greater relevance if we think that the interpretation of nature has changed substantially during the 20 th century. At present, it seems to be shaped by an environmen- 
tal awareness that has been increasing since the 1970s, reflected in publications such The Limits to Growth and the frequent use of terms as 'energy crisis' or 'climate change'. Thus, the vision of nature as something obvious, given, coherent and inexhaustible seems to have diminished. We are in a situation in which global perspectives have progressed considerably in our daily lives, being essential to think about the environmental consequences of our individual actions. In this sense, it seems opportune to ask about how to integrate these themes into our aesthetic conceptions.

If we observe the conception of nature throughout modernity, it has constantly developed the difference between preindustrial and industrial, between nature and the products manufactured by the human being, whether specific to the industry or any built environment. Cases such as the conception of Central Park by Frederick Law Olmsted are paradigmatic. In the text "Public Parks and the Enlargement of Towns" of 1870, Olmsted understands the park as something linked to the nature, as a focus of healthiness (alluding to the social hygiene movement), opposed to the city and the urban environment. This fact is also evidenced in Le Corubusier's urban projects, as Ville Radieuse (1922), where the housing towers spread out on uninterrupted nature. The fifth point for the new architecture also fosters this duality, conceived the garden terrace as return to nature the space occupied by building footprints.

But perhaps the case of the Crystal Palace is more manifest for what the building itself means for the modern conception. In this case, Joseph Paxton had to preserve a row of centenarian elms to achieve the permission, given by aristocratic Hyde Park neighbourhood, to carry out the pavilion for the 1851 London Great Exhibition. Thus, the building, which represents the advances of industrialization, both in the use of new materials (glass and steel) and in its own construction (assembled by an army of railway workers in four months), incorporates the nature that had rejected as an image (fig. 02).

The historian and critic Philip Ursprung calls to this attitude, or ideology, "naturalist", "on the assumption that there is actually such a thing as nature on the one hand, and that architecture's meaning resides in its capacity to articulate, frame, enhance and control the forces of nature". Ursprung proposes to leave this duality strengthen in modernity behind; "as the distinction between and wall and ceiling, inside and outside, become more difficult to make, the distinction between what is nature and what is not becomes obsolete". He adds: "nature and all the emblems it used to be represented by are totally absorbed into this new spatial realm"3.

If we pick up Robert Smithson's idea of nature as fiction, either from the notion of integration or from its existence as something independent, then we should reformulate the question of how nature affects and shapes the built environment. And, if we understand nature as an image, text or system of symbols, emerge also the possibility to transform and manipulate. Within this perspective, the idea of 'naturalisation' gets away from a conception of nature as something given or autonomous to the built environment, to assume it as a projection of the human being and consubstantial to the design itself.

The development of architecture from the conception of the nature itself, which lays out a specific visualization of this, is showed a way to explore, especially because the relevance that environmental awareness has acquired in recent decades. On the aesthetic dimensions of this perspective, Iñaki Ábalos points out that "only if there is genuine aesthetic debate, if there is an idea of beauty associated with sustainability, will the latter be able to appeal to architecture in a non-circumstantial way and will encourage architects to work on it". In his "Picturesque Atlas", Ábalos outlines a series of strategies to explore, drawing a narrative on parks and artificial landscapes, what is not known and its transformation into images ${ }^{4}$.

The idea of 'naturalisation' must be placed in this context and understood as a progress from those practices self-proclaimed sustainable or 'naturalist'. The latter seem to have polarized in two opposing strategies: one associated with the strictly technical and another with a kind of expressionism of natural aesthetics. In the first one, the architecture seems to be reduced to mere statistic, justifying any solution from the fulfilment of zero-energy building and to obtain the 'green building' certificate. And, on the other hand, we usually find a literal translation of natural forms, either through a green facade that envelops the building or converting the building directly into landscape. Unlike this view dominated by marketing that has made sustainability its all-purpose word, essentially of shortterm view, the following projects build a critical framework to delve into the relation between nature and architecture.

\section{NATURE AS METAPHOR: RENEW THE MODERN ARCHITECTURAL SPACE}

"With this simple construction, the Mediatheque will be the archetype of an entirely new architecture. It will serve as a place where the two bodies of the contemporary human being inhabit, the body that contains the flow of electrons and the primitive body responsive to nature"
The Sendai Mediatheque is the result of an international competition that led Toyo Ito to carry out, during 1995 and 2001, one of his most celebrated buildings. Its iconic image, the structural innovation or the programmatic flexibility give to the building an undoubted interest, but we can add the attempt to represent some topics of contemporary culture such as the fluidity condition, the functional multiplicity, the virtually of the limits or the advances of digital technology and electronic media. These topics have also been developed by Ito in various texts and articles in an effort to reflect on the changes of contemporary society.

Despite his awareness about the specificity of architecture recognizing its codes and a certain tradition, Ito considers essential finding in topicality part of the meaning of architecture (its contemporary condition). In order to introduce certain meanings in his work, the use of metaphor is frequent, as the Mediatheque exemplified, finding in the interpretation of nature or natural forms its main starting point. It should be noted that metaphor comes from the Greek term 'metaphora', which comes to say 'translation' or 'transfer', and which is based on the articulation of relationships of one thing with something different from itself to define a field of recognition and association as well as a transmission of certain meaning ${ }^{6}$.

The idea of column as a tree dominates the conception of the Mediatheque; while the building spatial definition seems to be based on the idea of forest, with a oscillation between open and close areas or lightness and darkness, setting up a varied composition (fig. 03). But atmospheric phenomena are also used in order to suggest an atmosphere composed of fluctuating activities; in this case, the information flows replace the natural ones. The diverse activities of each floor are conceived from this information exchange through conventional or micro-electronic systems. The multiplicity of layers (programs, or functions) or the phenomenalism completes this association, validating the idea that "architecture should not claim its own physical form but must become a device to interpret the form as a phenomenon"'.

In the article "Arquitectura pública como punto de paso" (or "The Transparent Urban Forest") Ito stands up for a city composed of transparent and permeable public buildings, as "relativized objects", with heterogeneous functions and spaces that can encourage the flows exchange (fig. 04). Following this principle, the Mediatheque is based on an open system of interchangeable activities trying to achieve a certain functional undefinedness. The integration of an art gallery, a municipal library and an audio-visual media centre seems analogous to an organic process of decomposition, combination or fusion.

In the Mediateque's formal description, understood as an archetype, Ito points out three kind of categories: plates (steel plates), tubes (steel structure) and skin (glass facade). It is referred, again, to the field of natural forms. The plates turn into a square steel slabs of $50 \times 50$ meters (seven), represented in the drawings as places that contain the flow of users and objects. It is favoured, thus, 
a system of variable densities able to guarantee a high diversity (the artificial lighting also acquires a important role in the exchange and variations of each floor) $)^{8}$. The section also contains this planimetric freedom through variation of heights between plates that accentuates the flexibility of the system. It should be added that the freedom achieved by Le Corbusier in the 'domino system' or Mies van der Rohe in the 'universal space', now is accompanied by a randomness character (fig. 05) ${ }^{9}$.

The Mediatheque's structure order and qualify the plan, since its strategic position in each platform guarantees the occupation and an adequate distribution of space. It is formed by 13 biomorphic tubes (like a trees), which penetrate vertically in the plates and support them. Each one, in addition to transport weight, allows the exchange of diverse energies, such as light, air, water or sound. The conception of the structure as something more than a bearing system is also observed in its ornamental dimension, leaves behind the conventions that insist on their independence. The structural function, thus, loses its foundation condition to camouflage itself in a decorative system defining environments and accentuating occupations.

Regarding the corporeity of architecture, the Mediatheque involves a tension between material requirements and the architect's desire to dilute building facade and its limits. The metaphor of the skin shows Ito's aspiration to turn the facade into an interface that could articulate the tangible and the intangible or the natural and the artificial. The real impossibility of this fact, since every architectural form or object is always based on a certain tectonic expression, reveals the limitations of such attempts. Ito, however, seems to delight in this ambiguity, hence his search for the virtual develops around his concerns for the tectonics and its use for metaphorical purposes.

The neutral and abstract space acting as a starting point at the Mediatheque, linked to the modern tradition, is altered through the recovering of architecture figurative quality and its literal and metaphorical ornamentation. This fact not only manifest that the paradox is assumed as a way of thinking, but also that the rejection of form could be compatible with techniques based on figure-ground contrast. At the same time, the use of metaphor by Toyo Ito emphasizes that nature not cease to be a cultural construct. Ito's deep interest in nature, as this project suggests, is not its contemplation or its hygienist values (the romantic and modern models) but its ability to allow us to interpret the cultural changes that the Digital era has introduced in our lives.

\section{NATURE AS ENERGY AND MEMORY: STRATIFICATION OF URBAN SPACE}

"On my first visit to the place, even with eighty cars parked there, I was touched by the beauty of the stone surface, the variety of sizes and colours of the material and the way that they shone in the sun. The physical history of Stortoget is based on the movement of these native stones from the neighbouring agricultural lands, where they were stacked forming boundaries for their use in the streets an the square at baroque town. The presence of this stone in Kalmar's centre recalls its physical transformation, almost primitive, from rural to urban area"10.

The Caruso St John's project, in collaboration with the Swedish artist Eva Löfdahl, had the task of renewing the main square of Kalmar in order to recover the old identity, lost over time and damaged by the traffic. Ruling out the strategy of tabula rasa, the authors conserve the existing fragmentation by restoring the patterns that had characterized the floor plan. This strategy leaves behind the artificial condition that can be perceived in many contemporary urban interventions, assuming the changes and the natural evolution of the urban space itself.

Caruso St John set out a plan based on two systems of order. On the one hand, a regulatory system through perpendicular routes to articulate flows and movements (built on smoother textures such as granite pavers and prefabricated concrete slabs); and, on the other hand, the specific definition of the areas generated by the first system through subtle changes in the stones and the granite's colour (granite boulders of different dimensions originally used in the square). If the first order gives unity to the square, the second allows an expressive richness in the floor's texture, which remembers the organic and heterogeneous condition of natural forms (fig. 06).

The intervention goes beyond the scope of the square itself. The definition of the layout system refers both to its perimeter and to the farthest urban environment, in order to integrate the square itself into the energy, cultural, and material network that appeals to the memory of the city. This definition of place, which seeks to broaden its interpretation as a mere typological interpretation, dissolves the permanence of the traces in a multiple relationship between the renewed space and the surroundings.

The project also seeks the abstraction of the floor plan, without fixing areas for specific uses. This polyvalence seems to assume the memory of the place, which has had multiples uses during its 300 years of existence - from political, military or religious representations to become the commercial heart of the city. In turn, any picturesque furniture or ornament is avoided emphasising the texture of the floor plan itself. Thus, the pavement acquires a clear physical presence, as an organic surface, thanks to the thickness and chiaroscuro of the stone patterns (fig. 07).

The matter becomes an active and sensitive object, a source of energy to deal with. The architects pay attention to this potential preserved in the displacements and the relocations of cobbles and boulders. The square organization finds in this matter's interpretation the identity of the place; a renewed genius loci. The possibility of understanding the square from its material memory assumes a reflective view of the natural and urban environment. The stone rests shifted and gathered, as natural entropic processes, making Stortorget a tangible sign of the city's life. As Adam Caruso points out: "We can also imagine the environment as something that can encompass human effort as well as matter, a territory in which connections can be established between energy and culture"11.

In Stortoget, also, urban space's idea is enriched through these connections between matter and energy, as layers of meaning that seeks, in any case, an architecture that could adopt a wider environmental sphere. The transport of stones from other places of the city to relocate within a new order is conceived as an operation where energy acquires it own expression and which is accumulated in a material that makes sensitive (fig. 08). This means an exchange of energy that enables to interpret the form, matter, memory or time within a process closely linked to the natural environment and its processes.

The Kalmar's stones, therefore, are shown as vehicle of energy and memory, which allow the past to emerge on the square surface, as layers of matter. All this within a process formed by stratums, which meaning is collected in the stones themselves. Time is interpreted as a discontinuous and non-linear variable, freed from ties to connect the experience of architecture with the place and life. The square, likewise, is revealed as a result of energy flows, recognizing a stratus of the past; sum of multiple levels that fixes a picture where the past and future are reduced to a specific present. We may well consider Stortoget as an instant place, whose granite stones, consist of energy and memory, dissolve between fleeting sparkles and water sounds.

\section{NATURE AS MEDIATION: THE PERCEPTION OF ATMOSPHERIC SPACE}

"Exercising the integration of the spectator, or, rather, the spectating itself, as part of the museum's undertaking has shifted the weight from the thing experienced to the experience itself. We stage the artefacts, but more importantly, we stage the way the artefacts are perceived. We cultivate nature into landscapes. So, to elude the museum's insistence that there is a nature (if you look hard enough for it), it is crucial not only to acknowledge that the experience itself is part of the process, but, more importantly, that experience must be presented undisguised to the spectator"12 
"The Mediated Motion" proposes a physical transformation of Kunsthaus Bregenz's architectural space through adding successive layers of nature that the viewers can contemplate in a continuous itinerary. The strict geometry of Peter Zumthor's building becomes in a kind of miniaturized but sublime nature and the museum in a sequence of atmospheric spaces that involve the visitors in its aesthetic density. The artistic efficiency of the installation is based on the immediate perceptive shock produced by the atmosphere of each landscape, which far from become a gimmicky effect, underlies a rigorous staging. The connexion between building and nature, also artificial, constitutes a dialogue where the natural seems to emanate from the artificial and the artificial from the natural.

The exposed work turns into nature and nature into landscape. The landscape architect Günther Vogt collaborates on the project, on the definition of the itinerary, based on the spiral movement that characterizes Zumthor's building, to emphasize the transition from one landscape situation to another. On the ground floor, visitors first encountered a collection of logs sprouting shiitake mushrooms. Moving to the levels above, they came across a pond with floating duckweed on level one, which they could cross via a series of pontoons, and a floor of gently sloping, compressed soil on level two. On the top floor, a suspension bridge spanning a room full of fog terminated abruptly at a blank wall, forcing visitors to return along their original route. The installation generates a tension between conceptual art, with scientific roots, and emotional one, subjective, based on the experience.

Eliasson considers mediation and movement -the two concepts selected to installation's title- essentials to delve into the relation between the human being and the environment. For the Danish artist, mediation supposes "a degree of representation in the experience of a situation"; degrees or levels in continuous changing according to the factors involved in each case. In this sense, mediation is understood from its capacity to generate evaluation, criticism, or reflection; key values of artistic and cultural practice. Regarding the movement, the installation emphasizes the idea of the travel and flow, whereby the spectator builds a critical attitude towards what is observed and perceived. The itinerary is not far from a funfair. The visitor is waiting the next room, expects the following trick (fig. 09). As Eliasson points out: "On all four levels, odours, fog, water, plants, and soil caused the strictly orthogonal, concrete-and-glass architecture of Peter Zumthor to metamorphose into a path of experience and awareness of experiencing"13.

The installation also aims to make us reflect on the weather and its capacity for mediation, understood as "nature in the city" whose interest lies in its unpredictability and variability. In the installation, Eliasson manipulates some basic elements of weather such as the water, light, temperature or atmospheric pressure itself. Through the natural phenomenon introduction, the installation promotes to think about how nature is perceived and how it affects in our perception of the environment; "As inhabitants, we have grown accustomed through our progressive experience of city space to the weather as mediated by the city. We experience the weather through the 'city-filter', as well as the other way around"14.

The use of nature goes with a process of dematerialization, where the atmospheres seem to dilute the limits that contain them. This accentuates the condition of the atmospheric model in which the exhibition aims to become, and encourages to reflect on the changing characteristics of architectural space. The light, projections or fog, used to manipulate the observer perception, provoke feelings that could be described as oneiric. At the same time, the perceptive mechanism tries to broaden those that act as only visual way to emphasize the non-visible ones. From this point of view, Eliasson defines an architectural reality where could be possible a deeper reflection on atmospheric space. These themes would be continued two years later in "The Weather Project", an installation held at the turbine hall of the Tate Modern in London (fig. 10) ${ }^{15}$.

Both installations emphasise the landscape's idea as a projected image of nature. At the Kunsthaus Bregenz and the Tate Modern, the relation between nature and architecture must be understood from the idea of mediation, where the visitor becomes the protagonist in the interaction with the artistic work. The Danish artist also gets away from the debate 'nature versus culture', to reconsider a field of experience constituted by both. Eliasson proposes that the nature should not be understood as a store of alternative forms to replace the conventional ones, but as energy source to activate our lives and as a backdrop to project them.

\section{Javier de Esteban}

Polytechnic University of Madrid. PhD in Architecture from the UPM (International Mention, 2016), MA in Architectural Design from ETSA Madrid (2011) and Architect from ETSA Universidad de Navarra. Assistant Professor in ETSA Valladolid (2016-2017) and Invited Lecturer in ETSAM Master in Advanced Design MPAA (2014-2017). He has been also Researcher in the Department of Architecture at University of Cambridge (2012-2013) and Assistant in the Research Group of Collective Housing (GIVCO, UPM). Partner of MydE Arquitectos.

\section{Notes}

01. ELIASSON, Olafur, "Seeing Yourself Sensing", in Leer es respirar, es devenir, Gustavo Gili, Barcelona, 2012, pp. 24.

02. SMITHSON, Robert, "A Museum of Language in the Vicinity of Art", Art International, march 1968. Also in FLAM, Jack, Robert Smithson: The Collected Writings, University of California Press, Berkeley, Los Ángeles, Londres, 1996.

03. URSPRUNG, Philip, Brechas y conexiones: ensayos sobre arquitectura, arte $y$ economía, Puente Editores, Barcelona, 2016.

04. The "Picturesque Atlas" leads the reader to the history of the nature's invention during nineteenth and twentieth centuries, in harmony with Robert Smithson's ideas. ABALOS, Iñaki, Atlas pintoresco. Vol. 1: el observatorio pintoresco. Vol. 2: los viajes. Gustavo Gili, Barcelona, 2005 y 2008. Also: ABALOS, Iñaki, "Thermodynamic beauty", in "Abalos+Sentkiewicz", 2G, 2010, n. 56, pp. 127-136.

05. ITO, Toyo, "Mediatheque in Sendai", in "Toyo Ito, Sección 1997", 2G, 1997, n. 2, pp. 26.
06. As the philosopher Mark Johnson points out, the metaphor may be understood as a mode of understanding through which certain fields of our experience are translated in order to structure another field of different type. JOHNSON, Mark, The body in the mind: the bodily basis of meaning, imagination, and reason, University of Chicago Press, Chicago, 1987, pp. XIII.

07. ITO, Toyo, "A Garden of Microchips. The Architectural Image of the Microelectronic Age", in Escritos, Colegio Oficial de Aparejadores y Arquitectos Técnicos de Murcia, Murcia, 2000, pp. 146-147.

08. As far as the artificial lighting is concerned, it is clearly visible in the building's images. The different heights of each floor acquire presence and formal and spatial relevance as a consequence of lighting variations - difference in intensity, in colour, or in warmth. These variations are responsible for the spatial diversities between floors, which constitutes a clearly anti-modern use of technology, considering that the representation through iconography is replaced by the spatial manipulation. 
09. ITO, Toyo, "La mediateca de Sendai. Informe sobre su proceso de construcción", in Escritos, Colegio Oficial de Aparejadores y Arquitectos Técnicos de Murcia, Murcia, 2000, pp. 229-230.

10. CARUSO, Adam, "La energía y la materia", in The feeling of things: escritos de arquitectura, Polígrafa, BarceIona, 2008, pp. 19-20.

11. lbid., pp. 15.

12. ELIASSON, Olafur, "Seeing Yourself Sensing", in Leer es respirar, es devenir, Gustavo Gili, Barcelona, 2012, pp. 24-25.

13. "In my search for clues to unlock the building -since the building is surely full of preconceptions of how to see and experience- I discovered the generous aspect of the spiral movement that takes you from one floor to the next. In order to take the greatest advantage of your tutored eye and to integrate you as a central player in the exhibition, I realised that enhancing the principle of movement would be the key. Since your movement and orientation is a process in time, I looked for an opportunity and medium to turn this particular process into the object. In one field in particular, the process as object has been cultivated -landscape architecturewhich is why I have turned to Günther Vogt, whose ideas on cultivating process have been a farsighted source for the development of this project: The mediated motion", ELIASSON, Olafur, "Dear Everybody", ibid., pp. 14.

14. Eliasson points out: "one might mistakenly take a situation for granted as a 'natural' state of things, being unaware of the constructions lying behind this situation. The challenge of orienting ourselves in a mediated realm is therefore to see through and know when, to what extent, and by whom a situation has been mediated; to be aware of a situation's relationship with time (...) When we can 'see through' the mediation of a situation, when it is transparent, we may experience a degree of heightened self-awareness due to the self-evaluative potential that lies within a situation like this", ELIASSON, Olafur, "Museums Are Radicals", Ibid., pp. 49.

15. In "The Weather Project", the representation of the sun and the sky dominates the space of the Turbine Hall in the Tate Modern. A fine fog expands through space as if it would have come from outside, varying during the day to simulate an atmospheric phenomena. Looking at the ceilings, the fog makes that the roof goes missing, replaced by the reflection of the floor. At the back of the room, we find a giant circle formed by hundreds of mono-frequency lamps. The repeated arc in the mirror produces a brilliant sphere linking the real space with the reflection itself. Generally used in public lighting, mono-frequency lamps emit a low-frequency light that mitigates all the colours except yellow and black. This installation transforms the visual field around the sun into a duotone landscape. PRIETO, Eduardo, "Ars meteorológica. Naturaleza y arquitectura en la obra de Olafur Eliasson", Arquitectura Viva, 2011, n. 141, pp. 74-75.

\section{Images}

01. Idea of city; where natural environment and built form an indivisible unit that favors the fluidity and interchangeability. El Croquis, Toyo Ito, 19 febrero 2011.

02. A) Villle Radieuse, Le Corbusier, 1922. B) Crystal Palace, Joseph Paxton, Gran Exposición de Londres de 1951.

03. Model, Sendai Media library, Toyo Ito, 1995-2001.

04. Idea of city; where natural environment and built form an indivisible unit that favors the fluidity and interchangeability. El Croquis, Toyo Ito, 19 febrero 2011.

05. Sketch of the plants and section, Model, Sendai Media library, Toyo Ito, 1995-2001.

06. Floor plan, Stortoget, Kalmar, Caruso St. John, 19992003.

07. Pavement detail, Stortoget, Kalmar, Caruso St. John, 19992003.

08. Stortoget, Kalmar, Caruso St. John, 1999- 2003.

09. "The Mediated Motion", 31.03-13.05 2001, Kunsthaus Bregenz, Olafur Eliasson.

10. A) "The Mediated Motion", 31.03-13.05, 2001, Kunsthaus Bregenz. B) "The Weather Project", 16.10.2003 - 21.03.2004, Tate Modern London. Olafur Eliasson. 